\title{
Sensing the lightness: a narrative analysis of an integrative medicine program for healthcare providers in the COVID-19 department
}

\author{
Eran Ben-Arye $^{1,2}$ (D) Shaked Zohar ${ }^{2} \cdot$ Yael Keshet $^{3} \cdot$ Orit Gressel $^{1,2} \cdot$ Noah Samuels $^{4} \cdot$ Arieh Eden $^{2,5} \cdot$ Jan Vagedes $^{6,7} \cdot$ \\ Sameer Kassem ${ }^{2,8}$
}

Received: 8 July 2021 / Accepted: 3 September 2021 / Published online: 15 September 2021

(c) The Author(s), under exclusive licence to Springer-Verlag GmbH Germany, part of Springer Nature 2021

\begin{abstract}
Objectives The research addressing physical and emotional exhaustion among healthcare providers (HCPs) in COVID-19 departments is limited. We examined the impact of integrative medicine (IM) intervention for HCPs working in isolated COVID-19 in-patient departments, addressing concerns and well-being.

Methods HCPs working in 3 isolated COVID-19 in-patient departments underwent 40-min IM treatment sessions (including acupuncture, manual movement, and/or mind-body modalities) provided by integrative oncology practitioners. The MYCAW (Measure Yourself Concerns and Well-being) questionnaire examined HCP concerns and free-text narratives following IM treatments. Data were qualitatively analyzed using ATLAS.Ti software for systematic coding.

Results A total of 181 HCPs underwent 305 IM treatments. Narrative themes focused on physical symptoms (primarily pain and fatigue) and emotional concerns, including perceived communication barriers with patients, and reflections on wellbeing and insights following IM treatments. HCPs reported feeling a sense of "relief" which was likely related to the 3 main effects of the IM intervention: a sense of "being cared for" and treated; experiencing emotional, sometimes spiritual effects of the treatment; and the feeling of relaxation, combined with the relief of pain. Qualitative analysis identified clusters of emotional and spiritual-related keywords such as "calming," "release," "relaxation," and "disengagement" following the first IM session (119 of 181 narratives, $65.7 \%$ ).

Conclusions HCPs working in isolated COVID-19 departments reported improved well-being and the addressing of their concerns following IM treatment sessions provided during their work shift. Further research is needed to explore the impact of IM on HCP burnout and resilience in palliative care settings.
\end{abstract}

Keywords COVID-19 $\cdot$ Integrative medicine $\cdot$ Pain $\cdot$ Burnout $\cdot$ Palliative care $\cdot$ Narrative-based medicine

Eran Ben-Arye and Shaked Zohar contributed equally to this study and are considered as co-first authors.

Eran Ben-Arye

eranben@ netvision.net.il

1 Integrative Oncology Program, The Oncology Service, Lin, Carmel, and Zebulun Medical Centers, Clalit Health Services, 35 Rothschild St., Haifa, Israel

2 Ruth and Bruch Rappaport Faculty of Medicine, Technion-Israel Institute of Technology, Haifa, Israel

3 Department of Sociology, Western Galilee Academic College, Galilee, Israel

4 Center for Integrative Complementary Medicine, Shaarei Zedek Medical Center, Faculty of Medicine, Hebrew University of Jerusalem, Jerusalem, Israel
5 Depatment of Anesthesiology, Critical Care and Pain Medicine, Lady Davis Carmel Medical Center, Haifa, Israel

6 ARCIM Institute, Research Institute, Filderstadt, Germany

7 Department of Neonatology, University Hospital Tuebingen, University Tuebingen, Tuebingen, Germany

8 Department of Internal Medicine, Lady Davis Carmel Medical Center, Haifa, Israel 


\section{Introduction}

Providing compassionate care to patients hospitalized in isolated COVID-19 departments is a challenging task for healthcare providers (HCPs) across the globe, with even the most experienced of professionals needing to increase resilience and overcome burnout and emotional exhaustion [1]. This has become evident in Wuhan, China, where the pandemic originated and reported among 52\% of the front-line nurses and over half of the physicians worldwide [2-4]. Working long shifts while wearing protective medical gear in isolation, with the constant need to monitor a patient's vital signs, have led to emotional distress manifest as fatigue [5], insomnia [6], anxiety [7], depression [8], fears of contagion [9], uncertainty [10], anger [11], and eventually leaving the COVID-19 department altogether [12].

Emotional distress and related concerns among HCPs working in COVID-19 departments are intensified by the overwhelming workload; difficulty in establishing open and direct communication with patients, many of whom are mechanically ventilated; professional frustration with the limited effectiveness of available treatments for COVID-19; the unpredictable nature of the disease, which can quickly deteriorate to life-threatening dyspnea; the ongoing risk to the staff (especially prior to the availability of vaccines); and, finally, a sense of isolation from colleagues and others from the outside who are unable to grasp the severity of their situation [13, 14]. In Argentina, researchers found that depersonalization had a mediating role between stress and hair cortisol levels among HCPs [15], though many report feelings of professional satisfaction, intensified by the gratitude of patients, informal caregivers, and the public, who often refer to them as "heroes" and "guardian angels [16].

In recognition of the need to address "burnout" among HCPs working in COVID-19 departments, the medical establishment has begun channeling resources to provide support. In Canada, HCPs reported lower levels of psychological distress, burnout, and plans to quit when it was perceived that resources such as counseling were made available [17]. HCP resilience was found to increase when effective leadership was in place, with greater support from peers [18]. There is also evidence that mind-body practices may reduce loneliness, stress, and anxiety, and improve sleep quality among physicians and advanced practice providers working in COVID-19 departments $[19,20]$.

In the present study, we explored the impact of an innovative integrative medicine (IM) intervention, with the goal of addressing concerns and improve well-being among HCPs and other personnel working in 3 COVID-19 in-patient hospital departments in a hospital in northern Israel. This paper presents the qualitative aspects of the IM program, based on HCP narratives. Future papers will present quantitative outcomes of the program, including physiological parameters.

\section{Methods}

\section{Study design and population}

This prospective, non-controlled study took place during the "third wave" of the COVID-19 pandemic in Israel (December 2020 to February 2021), in 3 isolated COVID-19 departments at the Carmel Medical Center in Haifa, Israel, with one designated as an intensive care unit (ICU). Eligible participants were aged $\geq 18$ years, working in the COVID-19 departments as HCPs (physicians and nurses from various hospital departments), consultant physicians (e.g., gynecologists, nephrologists), para-medical practitioners (e.g., physiotherapists, occupational therapists), technicians (e.g., respiratory, x-ray), and medical and adjuvant personnel (e.g., administration, cleaning).

\section{Referral to IM treatments}

Initially, the hospital administration reached out to HCPs working in the three COVID-19 departments, informing them of their eligibility to undergo IM treatments for quality of life (QoL) and well-being, and addressing workrelated concerns. IM sessions took place during work hours and were scheduled by either the HCP or department administration.

\section{Integrative treatment setting}

Treatment sessions were scheduled for $40 \mathrm{~min}$, beginning with a consultation with an integrative physician (IP), a medical doctor dually trained in integrative oncology and supportive care. The IP consultation included an in-depth explanation of the study protocol and following the signing of the study informed consent form the IP and HCP discussed the leading concerns and co-designed a treatment program. IM treatments were tailored in accordance with the HCP-reported concerns, lasting approximately $30 \mathrm{~min}$ and provided by an IM practitioner trained in at least two of the following modalities: acupuncture; touch (acupressure, anthroposophic medicine ointments, and reflexology) and movement (e.g., Feldenkrais and Paula methods, Qi Gong) therapies; and mind-body medicine (e.g., breathing, relaxation, and guided imagery).

All participating IM practitioners were working in an integrative oncology service, treating patients undergoing 
chemotherapy and palliative care at the Clalit Health Services Oncology Service (Lin, Zebulun, and Carmel medical centers, Haifa, Israel). The IM team included 2 integrative physicians, 2 integrative nurses, and 5 integrative therapists, all trained in supportive cancer care.

\section{Narrative assessment and analysis}

Pre- and post-assessment of the IM treatment program was conducted using the Measure Yourself Concerns and Wellbeing (MYCAW) questionnaire, a validated and reliable tool used in IM research [21]. MYCAW asks patients to list their two most significant concerns, scoring them from 0 (not bothering me at all) to 6 (bothers me greatly). Post-treatment MYCAW questionnaires include 2 additional open-ended questions which ask respondents to write a (from a few lines to several paragraphs) describing the most important aspects of their treatment. Numerical MYCAW scores have been shown to highly correlate with the Functional Assessment of Chronic Illness Therapy Questionnaire, including with the spiritual subscale (FACIT-SpEx), enabling more personal patient outcomes to be quantified, with qualitative coding considered to be generalizable across different integrative medicine settings [22]. Validated translations of the MYCAW questionnaires are available in Hebrew, Arabic, and Russian.

HCP interviews addressing MYCAW narratives from the open-ended questions were performed by integrative medicine practitioners, highly trained in interviewing patients and HCPs using the study tool in integrative oncology settings [23]. Qualitative analysis of the MYCAW narratives was conducted by qualitative research sociologists with extensive experience using this study tool within the context of an integrative medicine setting [24].

Free-text reflections reported by HCPs following the IM treatments were considered as short narratives, which were qualitatively analyzed using ATLAS.ti Scientific Software (V.8), enabling systematic coding. Qualitative content analysis was performed using a conventional content analysis approach, precluding the need for pre-established categories for coding [25, 26]. Short narratives were read line by line and codes were assigned to sections of the narratives, with codes representing core concepts discussed in each section. All quotes related to the codes were then gathered and sorted into categories on the basis of their relationship to each other and grouped into meaningful clusters with emerging themes.

\section{Ethical considerations}

The Ethics Review Board (Helsinki Committee) at the Carmel Medical Center in Haifa, Israel, approved and registered the study protocol (CMC-20-0202). It was deemed unethical to randomly allocate HCPs who were sacrificing their professional, personal, and physical wellness by their commitment to their patients to either true IM or control treatment groups. Participation in the study was voluntary, with no incentive offered such as payment or the like. All participating HCPs gave written consent.

\section{Results}

\section{Description of the study group}

The study group included $181 \mathrm{HCPs}$ and personnel working in the hospital COVID-19 departments. A total of 305 different IM treatments were administered during the study period (range, 1-8 sessions). In most sessions, a multi-modality IM treatment was provided. Manual-movement therapies were the most frequently used IM modality, followed by acupuncture, mind-body, and anthroposophic medicine (including the manual application of herbal ointments and oils). Participants were more likely to be female $(109,60.2 \%)$, of a median age of 34.5 years, with a predominance of nurses (90, 49.7\%) and physicians $(57,31.5 \%)$. The cohort was of cultural diversity, with Arabic the primary language (83, $47.7 \%$ ), and more than half reporting prior experience with complementary medicine therapies $(95,52.5 \%)$.

A total of 299 post-intervention MYCAW narratives were available for analysis.

\section{HCP concerns}

Many HCP narratives addressed the challenges faced by being on the "front line" in the isolated COVID-19 department. HCP concerns ranged from physical to emotional-spiritual domains and included the following identified themes:

\section{"Physical" themes}

These included the physical exertion required to work an entire shift wearing protective gear, the constant close contact with patients and the risk of infection (especially before the availability of effective vaccines; "It's a nightmare in there"). Other concerns included pain-related symptoms (reported in 94 narratives), intense fatigue (50), and gastrointestinal and appetite-related concerns (12 narratives). The pain was often described as "tightness" of the back, shoulder, and neck muscles or of an "overburdening" involving the back, head, neck, shoulders, shoulder blades, knees, and feet. Fatigue was often characterized as "total" and "overwhelming" and was associated with a feeling of "imbalance":

Unending fatigue...I lost my sense of balance...give me something to help me sleep. I never used to have 
any problem sleeping...How do I stop these thoughts? What is happening to me? (39-year-old male nurse who had been working for more than 9 months in the department).

An association was also made between fatigue and insomnia, felt to manifest as a sense of dissociation between mind and body:

The fatigue...I thought I had slept plenty, but it is never enough...the body sleeps; the mind does not (26-year-old nursing student, after 4 weeks in the COVID department).

\section{"Emotional" themes}

Emotional-related concerns were reported in many HCP narratives, some specifying emotional distress (37 narratives), primarily depression and anxiety-related symptoms; intense tension or stress (34); and concern for the health of family members (12). Emotional-psychological-spiritual concerns are described as a feeling of helplessness, frustration, depression, fear, and anxiety, all of which were exacerbated by the limited effectiveness of conventional medical treatments and the unpredictable course of illness:

I have no idea how...nothing seems to help...there is no drug, nothing... healthy people are dying... (30-year-old Arabic-speaking male physician, after 4 months of working in the COVID-19 department).

While some HCPs shared the feeling of "professional impotence," others felt the satisfaction of their professional role:

I finally feel that I am doing something. It's like being on the battlefield...everyone is going downhill. I enjoy being able to keep them alive. We have a shining team, a gathering of the exiles...top people... (49-year-old nurse "on loan" from the orthopedic department).

In many narratives, however, HCPs expressed excessive worrying, a feeling of being overworked, and low levels of energy. For 31 HCPs, the frustration at the inability to provide continuity of care was a major concern:

There is no continuity of care...it's all a big mess...we work from a gut feeling... we have no anchor. (31-yearold physician).

\section{Themes of somatization and transference}

Some HCPs were found to identify with the suffering of their patients, leading to somatization with the onset of physical symptoms such as dyspnea, which in 5 of the HCP narratives were described as intense and of both real and symbolic importance:

The image of patients hungry for air won't leave my head...I am hungry for air... (38-year-old senior nurse who had been working for 9 months in the COVID-19 ICU).

In some cases, the process of somatization made it difficult for HCPs to maintain emotional separation from their patients:

I become attached to the patient, despite trying not to be... (35-year-old Russian and Hebrew-speaking male nurse working in an internal medicine department).

A parallel process reported in HCP narratives was one of transference of concerns, from patients to HCPs:

My mood is low...sometimes crying, especially when someone dies in my arms...there is a great intensity at work...very tired... the anxiety of the patients is transferred to us... (48-year-old female physician working 50 weekly hours in the COVID-19 ICU).

\section{Post-treatment HCP reflections}

Most of the narratives were recorded immediately following the last IM treatment, some of which addressed previous sessions conducted in the COVID-19 department setting. The themes related to the HCPs' experience following IM treatments included the following:

\section{Sense of relief theme}

Three factors were found to be associated with this theme: The first was the sense of "being cared for" and treated and having been given time for themselves during the long and intense shifts. The second was the "emotional," possibly "spiritual experience" of the IM treatment program. The third was the "experience of relaxation," including the relief of pain, which allowed for the "release" of the body both physically and emotionally.

\section{Theme of "being care for"}

The therapeutic IM setting provided a "quiet and peaceful place...cutting off.. a moment to look after myself in the heavy daily workload" (32-year-old physician starting his internship, working 60 weekly hours in the COVID-19 department), and a sense of "being cared for" ("To feel that someone is treating me..."). A related theme was the appreciation by the HCPs that "someone else" was acknowledging their concerns and suffering: 
It's not taken for granted that you are here, and understand the pressure we are under...there's always this feeling that no one see us (29-year-old female intern).

The fact that IM treatments took place in the COVID-19 department, where HCPs were expected to play the role of "provider," had a significant non-specific therapeutic effect. This could be seen in the case of a 48 -year-old member of the cleaning staff, who wept from excitement following a combined touch and breathing therapeutic session:

I cry from excitement...I feel calm, my body rejuvenated...I felt your hands very well...like a mother giving to her son...after that, I no longer have any fear.

While some of the narratives described a feeling of distrust of the medical system and intense burnout, many saw the IM program as reflecting a genuine appreciation that "the system" was truly interesting in their well-being:

...that they are watching you and thinking about you and seeing you.... and that the department head screamed at me that I need to make time (to get the integrative treatment), and he was right...I have the strength now to return to work. (48-year-old physician).

\section{Moving through the emotional-to-spiritual continuum}

Many HCPs included emotional-spiritual (ES) keywords relating to calmness, "relaxation," "tranquility," "levity," "release," and "disengagement" (Table 1). The ES-related keywords were used significantly more frequently in the initial IM session (in 119 of 181 narratives; $65.7 \%$ ), less so in follow-up assessments, in $45.8 \%$ and $19.2 \%$ of the second and third sessions, respectively.

The ES keywords used following the first IM session were most often associated with a sense of relaxation, which contrasted with the stressful environment outside the hospital:
The treatment gives you a break, relaxation...I fell that I was able to release the constant pressure from work (49-year-old nurse recruited from the surgical department).

The "time-out" provided by the IM sessions allowed the HCPs to focus on themselves:

An opportunity to stop the events... a moment to focus on one's self...calm...returning to a place in your imagination (39-year-old female occupational therapist)

In some cases, the experience was accompanied by a sense of detachment, though in a positive context ("I went to a desert island... and cut off from everything") and an insight of release and "letting go" ("It's very calming... good for the soul... a moment like this for myself, my body, my soul... we forget ourselves"). A sense of levity, both emotional and physical ("a sense of lightness in the body"), was in stark contrast to the heaviness of the burden and the overwhelming responsibility of the COVID-19 department:

Reduced worries...disengaging from the surroundings...felt like all the heaviness is sinking (33-year-old male medical student, following his second IM treatment which included an anthroposophic medical oil massage, acupuncture, and guided imagery).

These sensations were associated with metaphors of enhanced vitality ("I received a boost of energy"), which occurred simultaneously with a sense of relaxation:

The treatment took me to another world after a few moments...a quiet, calm, relaxed world...a feeling of warm touch, felt like a magical hand which charged my empty battery with energy...the touch gave me energy...the quiet and the warmth which encompassed me gave me a feeling of floating in an empty space (a 45-year-old senior internist).
Table 1 Distribution of narrative keywords among COVID-19 healthcare practitioners undergoing integrative medicine interventions

\begin{tabular}{|c|c|c|c|}
\hline \multirow[t]{2}{*}{$\begin{array}{l}\text { Integrative medicine } \\
\text { session sequence }\end{array}$} & \multicolumn{3}{|l|}{$\begin{array}{l}\text { Entire HCP narratives } \\
N=299\end{array}$} \\
\hline & $\begin{array}{l}\text { Total number of HCP } \\
\text { narratives per session }\end{array}$ & $\begin{array}{l}\text { Number of narratives with } \\
\text { identified ES* keywords }\end{array}$ & $\begin{array}{l}\text { Number of narratives } \\
\text { with non-identified ES* } \\
\text { keywords }\end{array}$ \\
\hline 1st session & 181 & $119(65.7 \%)$ & $62(34.3 \%)$ \\
\hline 2nd session & 72 & $33(45.8 \%)$ & $83(54.2 \%)$ \\
\hline 3rd session & 26 & $5(19.2 \%)$ & $21(80.8 \%)$ \\
\hline$\geq 4$ th session & 20 & $5(25 \%)$ & $15(75 \%)$ \\
\hline All sessions & 299 & $162(54.2 \%)$ & $137(45.8 \%)$ \\
\hline
\end{tabular}

HCPs, healthcare practitioners

*ES (emotional and spiritual) includes HCP narratives with one or more of the following keywords: "calming," "release," "relaxation," and "disengagement" 
Other sensations included a dissociative experience, with "release" of the body from its constricted emotions:

I am calmer...I was released...the body was tensed up, so I let go organically and emotionally. I disengaged from the pressure (35-year-old physician).

This was expressed primarily with respect to pain-related concerns, for which the term "release" was used in a more physical context:

The touch released my neck...caused tingling in my hands...my neck is more free...I hope it lasts for a long time...it was more stiff...now much less... (30-year-old female radiology technician).

A 36-year-old female cleaning staff member, who was being treated with massage for the first time, described the transition of her pain from her "inside" to her "outside":

All the pain in my body was released through my feet". Others referred to their experience of pain: "I felt that the pain during the treatment was less severe...this is the first time in a long time that I couldn't remember that I had pain... (41-year-old nurse working $50 \mathrm{~h}$ weekly in the COVID department).

\section{Discussion}

The present study explored narratives of HCPs working on the "front line" of the isolated COVID-19 departments, who participated in an IM treatment program whose goal was to reduce their intensely physical and emotional distress. Quantitatively, pain and fatigue were the leading concerns reported by the HCPs. However, qualitative analysis suggests that emotional relief (based on the use of ES-related keywords) was a more common immediate outcome, most significantly following the initial IM treatment session. Both specific and non-specific effects may have also been related to the "mystery" of IM, with which the majority of the study HCPs was unfamiliar. This is in addition to being given a respite, even if only for $40 \mathrm{~min}$, from the intensely stressful and draining isolated environment of the COVID19 department. It was in these short minutes, being in their own "space," during which the HCPs could appreciate their own well-being, and not just that of their patients.

Identifying HCPs as "wounded healers" has long been understood to be an important step in ensuring the provision of care, especially in stressful situations where the suffering of patients is extreme, as seen in oncology care [27]. A similar impact on HCP mental health may also occur when treating severely ill COVID-19 patients [28]. Approaches to relieve HCP stress in this clinical setting include self-care
[29], psychosocial and mental health support [30], acupressure [31], yoga, and music therapy [32].

The qualitative analysis suggests that a multi-modal patient-tailored IM intervention may have alleviated emotional distress, inducing a short-term effect on HCPs' emotional experience following the IM intervention. The IM program was adapted from an ongoing integrative oncology setting, where both quantitative and qualitative pragmatic research assesses QoL and function of patients [33]. The main focus of both IM and palliative medicine, however, has been first and foremost to provide patient-centered care, with less emphasis on the needs of the HCPs responsible for this care. The present study suggests that IM should be further researched in the context of HCP wellness, including its potential to relieve burnout and compassion fatigue, while facilitating resilience and post-traumatic growth [34].

This study has a number of methodological limitations which need to be addressed in future research. A major study limitation is the decision to provide IM treatments to all HCPs working in the COVID-19 department, without a control group and with no randomization, most likely created a selection bias. And while all HCPs were encouraged to participate, there is no record of those who elected not to, precluding the generalizability of the findings. In addition, this qualitative study examined only self-reported HCP narratives, derived from the MYCAW open-ended questions which yielded a wide range of reflections, from few sentences to several paragraphs. Future research will need to employ the use of in-depth interviews. Another study limitation is the absence of objective outcome measures such as VAS scales to evaluate pre- and post-IM treatment symptom scores (e.g., pain, anxiety) and other physiologic parameters for stress reduction. The findings of the qualitative assessment may also reflect influencing factors, some non-specific (e.g., being taken care, quiet setting, rest) and some specific (direct effect on symptom severity). This creates a challenge in reaching conclusions regarding the extent to which the IM therapy was specific in addressing HCP concerns. The present study did not examine each of the 4 IM modalities individually, as most treatment sessions were multi-modal (e.g., acupuncture, anthroposophic manual therapy, and breathing exercise tailored during the intervention to the HCPs' expectations and concerns). In addition, the follow-up assessment of the period following the completion of the IM treatment program with qualitative outcomes examined only immediately reported narratives. Finally, the "generalizability" of the HCP-tailored IM program for other centers with diverse HCP populations remains to be shown.

In conclusion, the IM program described was created in response to the understanding that the intense and relentless "front-line" treatment of severely ill COVID-19 patients was taking its toll on the well-being and resilience of the HCPs working in these departments. The present qualitative study 
suggests that an IM treatment program provided (during work hours to HCPs working in isolated COVID-19 hospital departments is feasible and may induce a short-term beneficial effect on HCP's emotional-related concerns. Future research will need to explore the impact of the program on additional parameters such as job satisfaction, professional functioning in challenging clinical settings, prevention of burnout, and emotional exhaustion, enhancing the work environment and resilience among this HCP population.

Acknowledgements We are grateful to Dr. Avi Goldberg MD, director of Carmel Medical Center, and Shani Brosh, Ahuva Tal, and Keren Marom, for their unlimited support; the medical personnel of the three COVID-19 departments at the Carmel Medical Center; to Professor Elad Schiff, MD, and the integrative medicine team at the Bnai Zion Medical Center in Haifa, Israel, for sharing their insights regarding the pilot design of integrative medicine intervention in their hospital; to the integrative medicine teams at the Lin, Zebulun, and Carmel medical centers, who despite the risk agreed to treat patients and medical personnel at the COVID-19 departments: Dafna Wolf, Avigail Sagi, Sagi Shalev, Galit Galil, Dana Goldenblum, Raviv Peleg, and Meital Manches.

Author contributions E.B.A., S.Z., O.G., and S.K. organized the trial and collected the data analyzed in this study. E.B.A., O.G., and S.K. planned the study. E.B.A., Y.K., S.Z., and N.S. carried out the analysis and wrote a draft manuscript. All authors participated in the revision of the manuscript.

Availability of data and material Data transparency is available pending to request from the submitting author.

\section{Code availability N/A}

\section{Declarations}

Ethics approval The Ethics Review Board (Helsinki Committee) at the Carmel Medical Center in Haifa, Israel, approved and registered the study protocol (CMC-20-0202).

Consent to participate Participation in this study was voluntary and verified by participants' consent.

Consent for publication All authors consented to the publication of the present manuscript.

Conflict of interest The authors declare no competing interests.

\section{References}

1. Kok N, van Gurp J, Teerenstra S, van der Hoeven H, Fuchs M, Hoedemaekers C, Zegers M (2021) Coronavirus disease 2019 immediately increases burnout symptoms in ICU professionals: a longitudinal cohort study. Crit Care Med 49(3):419-427. https:// doi.org/10.1097/CCM.0000000000004865

2. Zhang X, Jiang X, Ni P, Li H, Li C, Zhou Q, Ou Z, Guo Y, Cao $\mathrm{J}$ (2021) Association between resilience and burnout of frontline nurses at the peak of the COVID-19 pandemic: positive and negative affect as mediators in Wuhan. Int J Ment Health Nurs. https://doi.org/10.1111/inm.12847

3. Asghar MS, Yasmin F, Alvi H, Shah SMI, Malhotra K, Farhan SA, Ali Naqvi SA, Yaseen R, Anwar S, Rasheed U (2021) Assessing the mental impact and burnout among physicians during the COVID-19 pandemic: a developing country single-center experience. Am J Trop Med Hyg. https://doi.org/10.4269/ajtmh.21-0141

4. Jalili M, Niroomand M, Hadavand F, Zeinali K, Fotouhi A (2021) Burnout among healthcare professionals during COVID-19 pandemic: a cross-sectional study. Int Arch Occup Environ Health 1-8. https://doi.org/10.1007/s00420-021-01695-x

5. Georger F, Dos Santos E, Gazagne L, Berdagué P, Saib A, Nahon S, Piquet J, Amara W (2020) COV IMPACT : analyse des différents facteurs de stress du personnel hospitalier dans 2 centres hospitaliers en France lors de la pandémie COVID-19 [COV IMPACT: Stress exposure analysis among hospital staff in 2 hospitals in France during the COVID-19 pandemic]. Ann Cardiol Angeiol (Paris) 69(5):227-232. French. https://doi.org/10.1016/j. ancard.2020.09.005.

6. Stewart NH, Koza A, Dhaon S, Shoushtari C, Martinez M, Arora VM (2021) Sleep in frontline healthcare workers during the COVID-19 pandemic: a social media survey study. J Med Internet Res. https://doi.org/10.2196/27331

7. Naldi A, Vallelonga F, Di Liberto A, Cavallo R, Agnesone M, Gonella M, Sauta MD, Lochner P, Tondo G, Bragazzi NL, Botto R, Leombruni P (2021) COVID-19 pandemic-related anxiety, distress and burnout: prevalence and associated factors in healthcare workers of North-West Italy. BJPsych Open 7(1):e27. https://doi. org/10.1192/bjo.2020.161

8. Denning M, Goh ET, Tan B, Kanneganti A, Almonte M, Scott A, Martin G, Clarke J, Sounderajah V, Markar S, Przybylowicz J, Chan YH, Sia CH, Chua YX, Sim K, Lim L, Tan L, Tan M, Sharma V, Ooi S, Winter Beatty J, Flott K, Mason S, Chidambaram S, Yalamanchili S, Zbikowska G, Fedorowski J, Dykowska G, Wells M, Purkayastha S, Kinross J (2021) Determinants of burnout and other aspects of psychological well-being in healthcare workers during the Covid-19 pandemic: a multinational cross-sectional study. PLoS ONE 16(4):e0238666. https://doi. org/10.1371/journal.pone.0238666

9. Zhang X, Jiang Y, Yu H, Jiang Y, Guan Q, Zhao W, Mao Y, Huang D, Hong W, Li D (2021) Psychological and occupational impact on healthcare workers and its associated factors during the COVID-19 outbreak in China. Int Arch Occup Environ Health 1-13. https://doi.org/10.1007/s00420-021-01657-3

10. Norful AA, Rosenfeld A, Schroeder K, Travers JL, Aliyu S (2021) Primary drivers and psychological manifestations of stress in frontline healthcare workforce during the initial COVID-19 outbreak in the United States. Gen Hosp Psychiatry 69:20-26. https:// doi.org/10.1016/j.genhosppsych.2021.01.001

11. Damico V, Murano L, Demoro G, Russello G, Cataldi G, D'Alessandro A (2020) Sindrome di Burnout tra il personale infermieristico italiano durante l'emergenza COVID 19. Indagine conoscitiva multicentrica [Burnout syndrome among Italian nursing staff during the COVID 19 emergency. Multicentric survey study]. Prof Inferm 73(4):250-257. Italian. https://doi.org/10. 7429/pi.2020.734250

12. Norkiene I, Jovarauskaite L, Kvedaraite M, Uppal E, Phull MK, Chander H, Halford K, Kazlauskas E (2021) 'Should I stay, or should I go?' Psychological distress predicts career change ideation among intensive care staff in Lithuania and the UK amid COVID-19 pandemic. Int J Environ Res Public Health 18(5):2660. https://doi.org/10.3390/ijerph18052660

13. Damico V, Murano L, Demoro G, Russello G, Cataldi G, D'Alessandro A (2020) Sindrome di Burnout tra il personale infermieristico italiano durante l'emergenza COVID 19. Indagine conoscitiva multicentrica [Burnout syndrome among Italian nursing 
staff during the COVID 19 emergency. Multicentric survey study]. Prof Inferm 73(4):250-257. Italian. https://doi.org/10.7429/pi. 2020.734250

14. Buran F, Altın Z (2021) Burnout among physicians working in a pandemic hospital during the COVID-19 pandemic. Leg Med (Tokyo) 51:101881. https://doi.org/10.1016/j.legalmed.2021. 101881

15. Ibar C, Fortuna F, Gonzalez D, Jamardo J, Jacobsen D, Pugliese L, Giraudo L, Ceres V, Mendoza C, Repetto EM, Reboredo G, Iglesias S, Azzara S, Berg G, Zopatti D, Fabre B (2021) Evaluation of stress, burnout and hair cortisol levels in health workers at a University Hospital during COVID-19 pandemic. Psychoneuroendocrinology 128:105213. https://doi.org/10.1016/j.psyneuen. 2021.105213

16. Marton G, Vergani L, Mazzocco K, Garassino MC, Pravettoni G (2020) 2020s Heroes Are are Not not Fearlessfearless: The the Impact impact of the COVID-19 Pandemic pandemic on Wellbeing wellbeing and Emotions emotions of Italian Health health Care care Workers workers During during Italy Phase phase 1. Front Psychol 11:588762. https://doi.org/10.3389/fpsyg.2020. 588762

17. Lou NM, Montreuil T, Feldman LS, Fried GM, Lavoie-Tremblay M, Bhanji F, Kennedy H, Kaneva P, Drouin S, Harley JM (2021) Evaluations of healthcare providers' perceived support from personal, hospital, and system resources: implications for wellbeing and management in healthcare in Montreal, Quebec, during COVID-19. Eval Health Prof. https://doi.org/10.1177/0163278721 1012742

18. Siddiqui I, Aurelio M, Gupta A, Blythe J, Khanji MY (2021) COVID-19: causes of anxiety and wellbeing support needs of healthcare professionals in the UK: a cross-sectional survey. Clin Med (Lond) 21(1):66-72. https://doi.org/10.7861/clinmed. 2020-0502

19. Thimmapuram J, Pargament R, Bell T, Schurk H, Madhusudhan DK (2021) Heartfulness meditation improves loneliness and sleep in physicians and advance practice providers during COVID-19 pandemic. Hosp Pract (1995). 1-9. https://doi.org/10.1080/21548 331.2021.1896858

20. Dincer B, Inangil D (2021) The effect of emotional freedom techniques on nurses' stress, anxiety, and burnout levels during the COVID-19 pandemic: a randomized controlled trial. Explore (NY) 17(2):109-114. https://doi.org/10.1016/j.explore.2020.11. 012

21. Paterson C, Thomas K, Manasse A, Cooke H, Peace G (2007) Measure Yourself Concerns and Wellbeing (MYCaW): an individualised questionnaire for evaluating outcome in cancer support care that includes complementary therapies. Complement Ther Med 15(1):38-45

22. Jolliffe R, Seers H, Jackson S, Caro E, Weeks L, Polley MJ (2015) The responsiveness, content validity, and convergent validity of the Measure Yourself Concerns and Wellbeing (MYCaW) patientreported outcome measure. Integr Cancer Ther 14(1):26-34. https://doi.org/10.1177/1534735414555809

23. Ben-Arye E, Dahan O, Shalom-Sharabi I, Samuels N (2018) Inverse relationship between reduced fatigue and severity of anemia in oncology patients treated with integrative medicine: understanding the paradox. Support Care Cancer 26(12):40394048. https://doi.org/10.1007/s00520-018-4271-5

24. Keshet Y, Schiff E, Samuels N, Ben-Arye E (2015) Giving voice to cancer patients: assessing non-specific effects of an integrative oncology therapeutic program via short patient narratives. Psychooncology 24(2):169-174. https://doi.org/10.1002/pon.3621

25. Keshet Y, Schiff E, Samuels N, Ben-Arye E (2015) Giving voice to cancer patients: assessing non-specific effects of an integrative oncology therapeutic program via short patient narratives. Psychooncology 24(2):169-174

26. Hsieh HF, Shannon SE (2005) Three approaches to qualitative content analysis. Qual Health Res 15(9):1277-1288

27. Gonzales M, Melton L (2017) The Wounded Healer. J Adv Pract Oncol 8(5):453-455

28. Ng QX, De Deyn MLZQ, Lim DY, Chan HW, Yeo WS (2020) The wounded healer: a narrative review of the mental health effects of the COVID-19 pandemic on healthcare workers. Asian J Psychiatr 54:102258. https://doi.org/10.1016/j.ajp.2020.102258

29. Waris Nawaz M, Imtiaz S, Kausar E (2020) Self-care of frontline health care workers: during COVID-19 pandemic. Psychiatr Danub 32(3-4):557-562. https://doi.org/10.24869/psyd.2020.557

30. Ripp J, Peccoralo L, Charney D (2020) Attending to the emotional well-being of the health care workforce in a New York City health system during the COVID-19 pandemic. Acad Med 95(8):11361139. https://doi.org/10.1097/ACM.0000000000003414

31. Dincer B, Inangil D (2021) The effect of emotional freedom techniques on nurses' stress, anxiety, and burnout levels during the COVID-19 pandemic: a randomized controlled trial. Explore (NY) 17(2):109-114. https://doi.org/10.1016/j.explore.2020.11. 012

32. Vajpeyee M, Tiwari S, Jain K, Modi P, Bhandari P, Monga G, Yadav LB, Bhardwaj H, Shroti AK, Singh S, Vajpeyee A (2021) Yoga and music intervention to reduce depression, anxiety, and stress during COVID-19 outbreak on healthcare workers. Int J Soc Psychiatry. https://doi.org/10.1177/00207640211006742

33. Ben-Arye E, Elly D, Samuels N, Gressel O, Shulman K, Schiff E, Lavie O, Minerbi A (2021) Effects of a patient-tailored integrative oncology intervention in the relief of pain in palliative and supportive cancer care. J Cancer Res Clin Oncol. https://doi.org/ 10.1007/s00432-020-03506-1

34. Koh MYH, Hum AYM, Khoo HS, Ho AHY, Chong PH, Ong WY, Ong J, Neo PSH, Yong WC (2020) Burnout and resilience after a decade in palliative care: what survivors have to teach us. A qualitative study of palliative care clinicians with more than 10 years of experience. J Pain Symptom Manage. 59(1):105-115. https://doi.org/10.1016/j.jpainsymman.2019.08.008

Publisher's note Springer Nature remains neutral with regard to jurisdictional claims in published maps and institutional affiliations. 